\title{
A Product Integration Approach Based on New Orthogonal Polynomials for Nonlinear Weakly Singular Integral Equations
}

\author{
M. Rasty • M. Hadizadeh
}

Received: 23 July 2008 / Accepted: 22 October 2008 / Published online: 30 October 2008

(C) Springer Science+Business Media B.V. 2008

\begin{abstract}
This paper provides with a generalization of the work by Chelyshkov (Electron. Trans. Numer. Anal. 25(7): 17-26, 2006), who has introduced sequences of orthogonal polynomials over $[0,1]$ which can be expressed in terms of Jacobi polynomials. We develop a new approach of product integration algorithm based on these orthogonal polynomials including the numerical quadratures for solving the nonlinear weakly singular Volterra integral equations. The convergence analysis of the proposed scheme is derived and numerical results are given showing a marked improvement in comparison with recent numerical methods.
\end{abstract}

Keywords Nonlinear weakly singular integral equation · Product integration · Orthogonal polynomials $\cdot$ Convergence analysis $\cdot$ Numerical treatments

Mathematics Subject Classification (2000) Primary 65R20 · Secondary 45E10

\section{Introduction}

Integral equations of Volterra type with weakly singular kernels arise in many modelling problems in mathematical physics and chemical reactions, such as stereology [16], heat conduction, crystal growth, electrochemistery, superfluidity [15], the radiation of heat from a semi infinite solid [11] and many other practical applications. We remark here that equations of this type have been the focus of many papers $[5,7,10,17,22,24,25]$ in recent years.

In this paper we consider the nonlinear Volterra integral equations of the second kind,

$$
f(x)=g(x)+\int_{0}^{x} p(x, t) K(x, t, f(t)) d t, \quad x \in[0, T]
$$

M. Rasty $\cdot$ M. Hadizadeh $(\bowtie)$

Department of Mathematics, K.N. Toosi University of Technology, Tehran, Iran

e-mail: hadizadeh@kntu.ac.ir

M. Rasty

e-mail: mrasti@sina.kntu.ac.ir 
where the kernel $p(x, t)$ is weakly singular and the given functions $g$ and $K$ are assumed to be sufficiently smooth in order to guarantee the existence and uniqueness of a solution $f(x) \in C[a, b]$ (see for instance, $[1,4]$ ). Typical forms of $p(x, t)$ are

(i) $p(x, t)=|x-t|^{-\alpha}, 0<\alpha<1$,

(ii) $p(x, t)=\log |x-t|$.

It is well known that for Volterra equations with bounded kernels, the smoothness of the kernel and of the forcing function $g(x)$ determines the smoothness of the solution on the closed interval $[0, X]$, with $X>0$. If we allow weakly singular kernels, then the resulting solutions are typically non smooth at the initial point of the interval of integration, where their derivatives become unbounded. Some results concerning the behavior of the exact solution of equations of type (1.1) are given in [4].

The numerical solvability of weakly singular integral equations and other related equations have been pursued by several authors. The existence and uniqueness results of solution (1.1) have been proved in [12] by Kershaw using the Banach's fixed point theorem. Moreover, using Lagrange linear interpolation formula, he presented a collocation method for solving (1.1) and proved convergence of the approximated solution, but the order of convergence is very low. Due to unboundedness of the first derivative $f^{\prime}(x)$ of the solution $f(x)$ in (1.1) at the initial points of the interval of integration there has been difficulties for numerical treatment of (1.1). Brunner in [3] pointed out under quasi-uniform meshes the order of convergence of any polynomial spline collocation approximation is only $1-\alpha$. Although, he proved that under a suitably graded mesh the polynomial spline collocation approximation with the degree $m$ may theoretically obtain the order $m$ of accuracy, the optimal order cannot be obtained because of serious round-off errors. Wazwaz and Khuri [25] solved (1.1) by using the Adomian decomposition method and the phenomenon of self-cancelling "noise" term. Also, the piecewise polynomial collocation scheme has been proposed by Brunner et al. in [5]. Recently, Khater et al. [10] have been concerned with the method of one step $v$-stage Chebyshev expansion for the logarithmic singularities of (1.1).

On the other hand, quadrature methods without doing any computations of integrals for getting the weight coefficients, have been effectively applied to solve Volterra integral equations with some smooth kernels, but there are only few papers dealing with these methods to solving nonlinear weakly singular Volterra integral equations. In [23] Tao and Yong presented a new quadrature method for solving (1.1) based on Navot's quadrature rule and proved that the error is of order $O\left(h^{2-\alpha}\right)$. Also, the same authors focused on direct quadrature methods and their extrapolation for solving (1.1) in [24]. Orsi [21] proposed to apply product integration method to solve linear form of (1.1), where a Nystrom method is used on a small interval $[a, c]$ and a step by step method is used on $[c, b]$. Baratella and Orsi in [2] used Simpson's product integration scheme to solve a linear form of (1.1) and reached good results. In the mentioned works the authors have been used a transformation that a linear weakly singular Volterra integral equation of the second kind can be transformed into an equation which is still weakly singular, but whose solution is as smooth as we like.

In this paper, we provide a new strategy of product integration algorithm for numerical solution of weakly singular equation (1.1). The offered disceretization scheme uses Gaussian quadratures based on the new orthogonal polynomials which proposed to [6]. Using the roots of new orthogonal polynomials which will be introduced, we will show that a more exact quadrature can be obtained by which the integral part of (1.1) can be well-approximated even in the nonlinear case.

The layout of this paper is as follows. In Sect. 2 we are briefly introduced the new families of orthogonal polynomials and establish a Gauss-type numerical quadrature which has 
presented in [6]. In Sect. 3 we describe an application of the quadrature method obtained in Sect. 2 as a product integration scheme for numerical solution of nonlinear weakly singular Volterra integral equations. In Sect. 4 the convergence analysis of the proposed method is investigated and finally in Sect. 5 some numerical experiments are reported to clarify the method and some comparisons are made with existing methods in the literature.

\section{Basic Concepts and Preliminaries}

Recently, Chelyshkov has introduced sequences of polynomials in [6], which are orthogonal over the interval $[0,1]$ with the weight function 1 . These polynomials are explicitly defined by

$$
P_{N k}(x)=\sum_{j=0}^{N-k}(-1)^{j}\left(\begin{array}{c}
N-k \\
j
\end{array}\right)\left(\begin{array}{c}
N+k+1+j \\
N-k
\end{array}\right) x^{k+j}, \quad k=0,1, \ldots, N .
$$

The polynomials $P_{N k}(x)$ have properties, which are analogous to the properties of the classical orthogonal polynomials. These polynomials can also be connected to a fixed set of Jacobi polynomials $P_{m}^{(\alpha, \beta)}(\xi)$. Precisely

$$
P_{N k}(x)=x^{N} P_{k-N}^{(2 N, 0)}(1-2 x) .
$$

In this section, the numerical quadrature based on the new orthogonal polynomials will be described.

Investigating more on (2.1), we deduce that in the family of orthogonal polynomials $\left\{P_{N k}(x)\right\}_{k=0}^{N}$ every member has degree $N$ with $N-k$ simple roots. Hence, for every $N$ the polynomial $P_{N 0}(x)$ has exactly $N$ simple roots in $(0,1)$. Following [6], it can be shown that the sequence of polynomials $\left\{P_{N 0}(x)\right\}_{N=0}^{\infty}$ generate a family of orthogonal polynomials on $[0,1]$ which possesses all the properties of other classic orthogonal polynomials e.g. Legendre or Chebyshev polynomials. Therefor, if the roots of $P_{N 0}(x)$ are chosen as node points, then we can obtain an accurate numerical quadrature.

We reassemble in the following theorem some of the results obtained in [6], which determines exactness of the quadrature.

Theorem 1 (From [6]) The quadrature

$$
\int_{0}^{1} f(x) d x \approx w_{0} f(0)+\sum_{j=1}^{N} w_{j} f\left(x_{j}\right)
$$

is exact for any polynomial of degree $\leq 2 N$ iff $x_{j}$ are the zeros of the polynomial $P_{N 0}(x)$ in $(0,1)$ and weighting coefficients $w_{j},(j=1, \ldots, N)$ as follows

$$
w_{j}=-\frac{2}{N(N+1)(N+2)} \frac{\sum_{k=1}^{N}(2 k+1) P_{N k}\left(x_{j}\right)}{x_{j}^{2} P_{N 1}(x) P^{\prime} N 0\left(x_{j}\right)},
$$

and $w_{0}=1-\sum_{j=1}^{N} w_{j}$

This result generalize the exactness of the quadrature respect to the classical Gaussian quadrature schemes e.g. Gauss-Legendre, Gauss-Chebyshev and etc. 


\section{The Algorithm}

Before describing the disceretization algorithm, it is useful to recall the product integration scheme which can be used to approximate integrals of the form

$$
\int_{0}^{x} p(x, t) K(x, t, f(t)) d t
$$

where $p(x, t)$ is the weakly singular kernel of type (i) or (ii). If we use the $N$-point quadrature rule and collocate (1.1) at the nodes $\left\{x_{i}\right\}_{i=1}^{N} \bigcup\{0\}$, we get

$$
f\left(x_{i}\right)=g\left(x_{i}\right)+\int_{0}^{x_{i}} p\left(x_{i}, t\right) K\left(x_{i}, t, f(t)\right) d t, \quad i=0,1, \ldots, N, x_{0}=0 .
$$

For approximating the integral term, where $p(x, t)$ is a weakly singular kernel, we use the Lagrange interpolating polynomial to approximate $K\left(x_{i}, t, f(t)\right)$

$$
L_{N}(K ; t)=\sum_{j=0}^{N} l_{N j}(t) K\left(x_{i}, x_{j}, f\left(x_{j}\right)\right), \quad i=0,1, \ldots, N, x_{0}=0,
$$

with

$$
l_{N j}(x)=\prod_{j=0, j \neq i}^{N} \frac{\left(x-x_{j}\right)}{\left(x_{i}-x_{j}\right)}, \quad i=0,1, \ldots, N, x_{0}=0 .
$$

Defining

$$
w_{i j}=\int_{0}^{x_{i}} p\left(x_{i}, t\right) l_{N j}(t) d t, \quad i, j=1,2, \ldots, N,
$$

we can approximate the integral by

$$
\int_{0}^{x_{i}} p(x, t) K(x, t, f(t)) d t=\sum_{j=0}^{N} w_{i j} K\left(x_{i}, t_{j}, f\left(t_{j}\right)\right), \quad i=1,2, \ldots, N .
$$

\subsection{Discretization}

Following the product integration scheme, the Nystrom method on grids $\left\{x_{i}\right\}_{i=1}^{N} \bigcup\{0\}$ gives

$$
f\left(x_{i}\right)=g\left(x_{i}\right)+\sum_{j=0}^{N} w_{i j} K\left(x_{i}, t_{j}, f\left(t_{j}\right)\right), \quad i=0,1, \ldots, N,
$$

where $\left\{x_{i}\right\}_{i=1}^{N}$ are the roots of $N^{t h}$ degree polynomial $P_{N 0}(x)$ and $w_{i j}$ are the weight coefficients which can be obtained from (3.1).

Note that the relation $(3.3)$ is a $(N+1) \times(N+1)$ nonlinear system of equations which has a unique solution (see e.g. [9, 24]). Solving this nonlinear system determines the values of $f\left(t_{i}\right)$ for $i=0,1, \ldots, N$ which are the solutions of (1.1) in the points $\left\{x_{i}\right\}_{i=1}^{N} \bigcup\{0\}$.

In order to obtaining the numerical solution in any arbitrary point $\xi$ in the $[0,1]$, we are concerned with a rule which is depended on $w^{*}$ and $f(\xi)$ as follows

$$
\int_{0}^{1} f(x) d x \approx \sum_{j=0}^{N} w_{j} f\left(x_{j}\right)+w^{*} f(\xi) .
$$


Clearly, this quadrature is exact for polynomials of degree $\leq 2 N[13,14]$. However, in the case of quadrature rules which include among their nodes, in addition the point $\xi$ as a collocation point, we will obtain $(N+2) \times(N+2)$ nonlinear system of equations whose solutions give the value of any our grid points especially at the point $x_{N+1}=\xi$. In Sect. 5 we will set $x_{N+1}=1$ only for comparing the numerical results and it can be substituted by any value in $(0,1]$.

\section{Convergence Analysis}

In our convergence analysis we examine the linear test equation

$$
f(x)=g(x)+\int_{0}^{x} p(x, t) f(t) d t, \quad 0 \leq x \leq 1,
$$

and assume that the function $g(x) \in C[0,1]$, and the kernel $p(x, t)$ is weakly singular of the form (i) or (ii). In this case, (4.1) has a unique solution $f(x) \in C[0,1]$ that may be expected to have unbounded derivatives at the endpoints.

The grid points $\left\{x_{i}\right\}_{i=1}^{N} \bigcup\{0\}$ including the described disceretization give rise

$$
f_{N}\left(x_{j}\right)=g\left(x_{j}\right)+\sum_{j=0}^{N} w_{j}(p ; x) f_{N}\left(x_{j}\right)
$$

where $x_{0}=0$.

In order to examine the uniform convergence of the approximate solution $f_{N}(x)$ to the exact solution $f(x)$ of (4.1), notice that

$$
f(x)-f_{N}(x)=\sum_{j=0}^{N} w_{j}(p ; x)\left\{f\left(x_{j}\right)-f_{N}\left(x_{j}\right)\right\}+t_{N}(p, f ; x),
$$

where $t_{N}(p, f ; x)$ is the local truncation error defined by

$$
t_{N}(p, f ; x)=\int_{0}^{x} p(x, t) f(t) d t-\sum_{j=0}^{N} w_{j}(p ; x) f\left(x_{j}\right) .
$$

Let $A_{N}$ be the linear operator from $C[0,1]$ into $C[0,1]$, which is defined by

$$
A_{N} f(x)=\sum_{j=0}^{N} w_{j}(p ; x) f\left(x_{j}\right), \quad f \in C[0,1], x \in[0,1],
$$

then

$$
\begin{aligned}
\left\|f(x)-f_{N}(x)\right\|_{\infty} & \leq\left\|A_{N} f-A_{N} f_{N}\right\|_{\infty}+\left\|t_{N}\right\|_{\infty} \\
& \leq\left\|A_{N}\right\|_{\infty}\left\|f(x)-f_{N}(x)\right\|_{\infty}+\left\|t_{N}\right\|_{\infty}
\end{aligned}
$$

and by this terminology and considering (4.2), we will obtain

$$
\left\|f(x)-f_{N}(x)\right\|_{\infty} \leq\left\|\left(I-A_{N}\right)^{-1}\right\|_{\infty}\left\|t_{N}\right\|_{\infty} .
$$


Our final goal is to determine an upper bound for $\left\|f(x)-f_{N}(x)\right\|_{\infty}$. For this purpose, firstly we recall the following auxiliary lemmas regarding to kernels of type (i) from [8]. Throughout this section, the symbol const stands for a positive constant taking different values on different occurrences.

Lemma 1 (From [8]) The integral

$$
\int_{a}^{1}\left(\sqrt{1-x}+N^{-1}\right)^{\lambda}|x-t|^{v} d x
$$

with $-1 \leq a<1, \lambda<0, v>-1$ and $|t| \leq 1$, admits the following bounds

$$
\int_{a}^{1}\left(\sqrt{1-x}+N^{-1}\right)^{\lambda}|x-t|^{v} d x \leq \mathrm{const} \begin{cases}1, & \lambda / 2+v+1>0 \\ \log N, & \lambda / 2+v+1=0 \\ N^{-2-2 \lambda-2 v}, & \lambda / 2+v+1<0 .\end{cases}
$$

Lemma 2 (From [8]) Let $\sigma$ and $v$ be real numbers with $\sigma, v>-1, \sigma+v>-1$ and $\sigma$ not a integer. Given any positive integer $s$, there is an algebraic polynomial $Q_{N}$ of degree $N$ such that

$$
\left|(1-x)^{\sigma}-Q_{N}(x)\right| \leq \frac{\text { const }}{N^{s+\sigma+1}}(1-x)^{\sigma / 2-(s+1) / 2}(1+x)^{\sigma / 2+(s+1) / 2}, \quad 0 \leq x<1,
$$

moreover

$$
\int_{0}^{1}\left|(1-x)^{\sigma}-Q_{N}(x)\right||x-t|^{v} d x \leq \mathrm{const} \begin{cases}N^{-2-2 \sigma-2 v}, & |t| \leq 1, v<0 \\ N^{-2-2 \sigma}, & |t| \leq 1, v \geq 0\end{cases}
$$

where const is independent of $t$ and $N$.

Note that there are similar lemmas for kernels of type (ii) (e.g. logarithmic kernels) in [8]. Now, we define the following local error functions for the approximate solution of (3.2)

$$
R_{N}(f, t)=\int_{0}^{1} K(x, t) f(x) d x-I_{N}(f, t)
$$

with

$$
I_{N}(f, t)=\int_{0}^{1} K(x, t) L_{N}^{*}(f ; x) d x=\sum_{j=0}^{N} w_{j} f\left(x_{j}\right),
$$

where $L_{N}^{*}(f ; x)$ is the Lagrange interpolation polynomial which interpolates $f(x)$ in the points $\left\{x_{i}\right\}_{i=1}^{N} \bigcup\{0\}$, and is given by

$$
L_{N}^{*}(f ; x)=\sum_{i=0}^{N} f\left(x_{i}\right) l_{N j}(x), \quad x_{0}=0
$$

and the fundamental polynomial $l_{N j}(x)$ is in the form

$$
l_{N j}(x)=\prod_{i=0, i \neq j}^{N} \frac{\left(x-x_{j}\right)}{\left(x_{i}-x_{j}\right)}, \quad j=0, \ldots, N, x_{0}=0 .
$$


Following [20], we know that $R_{N}(f)=O\left(N^{-n}\right)$ where $f \in C^{n}[0,1]$. Now, suppose that the function $f$ be a weakly singular function in endpoints, e.g. in $x=1$, it means $f(x)=(1-x)^{\sigma}, \sigma>-1$. It is clear that if $f$ be a polynomial of degree $N$ then $R_{N}(f, t)=0$, so as defined in [8], we can write for all polynomials $P_{N}$ of degree $N$

$$
R_{N}(f, t)=\int_{0}^{1} K(x, t)\left[f(x)-P_{N}(x)\right] d x-\int_{0}^{1} K(x, t)\left[L_{N}^{*}\left(f-P_{N}\right) ; x\right] d x .
$$

By a proper choice of the sequence of polynomials $\left\{P_{N}\right\}$, we will be able to derive an upper bounds for the two terms

$$
\begin{aligned}
& R_{N}^{(1)}(f, t)=\int_{0}^{1}|K(x, t)|\left|f(x)-P_{N}(x)\right| d x, \\
& R_{N}^{(2)}(f, t)=\int_{0}^{1}|K(x, t)|\left|L_{N}^{*}\left(f-P_{N} ; x\right)\right| d x,
\end{aligned}
$$

where $K(x, t)$ is the weakly singular kernel of types (i) or (ii).

We are now ready to prove the following theorem that is our major equipment to find an upper bound for the truncation error in (4.4).

Theorem 2 Let $f(x)=(1-x)^{\sigma}, \sigma>-1$ (not integer) and $v>-1$, with $\sigma+v>-1$, then

$$
\int_{0}^{1}\left|f(x)-L_{N}^{*}(f ; x)\right||x-t|^{v} d x \leq \mathrm{const} \begin{cases}N^{-2-2 \sigma-2 v} \log N, & |t| \leq 1, v<0 \\ N^{-2-2 \sigma} \log N, & |t| \leq 1, v \geq 0\end{cases}
$$

where const is independent of $t$ and $N$.

Proof Let $Q_{N}$ be the polynomial which satisfies in Lemma 2 and consider the inequality

$$
\begin{aligned}
& \int_{0}^{1}\left|f(x)-L_{N}^{*}(f ; x)\right||x-t|^{v} d x \\
& \quad \leq \int_{0}^{1}\left|f(x)-Q_{N}(x)\right||x-t|^{v} d x+\int_{0}^{1}\left|L_{N}^{*}\left(f-Q_{N} ; x\right) \| x-t\right|^{v} d x \\
& \quad=I_{1}(t)+I_{2}(t) .
\end{aligned}
$$

Lemma 1 insures that $I_{1}(t)$ is bounded, while for $I_{2}(t)$ we need some more computations. Now, set $r_{N}=f-Q_{N}$, hence

$$
\left|L_{N}^{*}\left(r_{N} ; x\right)\right| \leq\left|l_{N}\left(x_{c}\right)\right|\left|r_{N}\left(x_{c}\right)\right|+\sum_{k=0, k \neq c}^{N}\left|l_{N}\left(x_{k}\right)\right|\left|r_{N}\left(x_{k}\right)\right|,
$$

where $c$ is the index corresponding to the closest knot to $x$. Due to Theorem 33 of [19], we have

$$
\left|l_{N}\left(x_{c}\right)\right| \leq \text { const }, \quad 0 \leq x \leq 1,
$$

furthermore, relations (4.8), together inequalities (1), (14) and (20) in [20], give us

$$
\left|l_{N}\left(x_{k}\right)\right| \leq \mathrm{const} \frac{\left(1-x_{k}\right)^{3 / 4}\left(1+x_{k}\right)^{3 / 4}\left|P_{N 0}(x)\right|}{N\left|x-x_{k}\right|}, \quad k \neq c,
$$


where $P_{N 0}(x)$ is the $N^{\text {th }}$ degree member of a family of new orthogonal polynomials. Taking account (4.5), we deduce for any integer $s \geq 1$

$$
\begin{aligned}
\left|L_{N}^{*}\left(r_{N} ; x\right)\right| \leq & \text { const }\left\{\frac{\left(1-x_{c}\right)^{(\sigma-s) / 2}}{N^{\sigma+s}}+\frac{(1+x)\left|P_{N 0}(x)\right|}{N^{\sigma+s}}\right. \\
& \left.\times \sum_{k=0, k \neq c}^{N} \frac{\left(1-x_{k}\right)^{\sigma / 2+3 / 4-s / 2}\left(1+x_{k}\right)^{\sigma / 2+3 / 4+s / 2-1}}{N\left|x-x_{k}\right|}\right\} .
\end{aligned}
$$

We will make use of some results of Nevai [20], which state the necessary and sufficient conditions for weighted mean convergence of Lagrange interpolation based at zeroes of generalized Jacobi polynomials. Actually, as stated in [20, p. 673] the following relations for $x_{c}$ and $P_{N 0}(x)$ hold

$$
\begin{aligned}
1-x_{c} & \geq \operatorname{const}\left(\sqrt{1-x}+N^{-1}\right)^{2}, \\
\left|P_{N 0}(x)\right| & \leq \operatorname{const}\left(\sqrt{1-x}+N^{-1}\right)^{-1 / 2}\left(\sqrt{1+x}+N^{-1}\right)^{-1 / 2} .
\end{aligned}
$$

Based on these relations, (4.9) can be rewritten as

$$
\begin{aligned}
\left|L_{N}^{*}\left(r_{N} ; x\right)\right| \leq & \frac{\text { const }}{N^{s+\sigma}}\left\{\left(\sqrt{1-x}+N^{-1}\right)^{\sigma-s}+\left(\sqrt{1-x}+N^{-1}\right)^{-1 / 2}\right. \\
& \left.\times\left(\sqrt{1+x}+N^{-1}\right)^{3 / 2} \sum_{k=1, k \neq c}^{N} \frac{\left(1-x_{k}\right)^{\sigma / 2+3 / 4-s / 2}}{N\left|x-x_{k}\right|}\right\} .
\end{aligned}
$$

In virtue of the first equation of (3.6) in Lemma 5 of [8] for $s>\sigma+5 / 2$, which finds an upper bound for the latter summation, we have

$$
\begin{aligned}
\left|L_{N}^{*}\left(r_{N} ; x\right)\right| \leq & \text { const }\left\{\frac{\left(\sqrt{1-x}+N^{-1}\right)^{3 / 2}}{N^{s+\sigma}}\right. \\
& \left.\times\left[\frac{\left(\sqrt{1-x}+N^{-1}\right)^{-5 / 2}}{N^{\sigma+5 / 2-s}}+\left(\sqrt{1-x}+N^{-1}\right)^{\sigma-s} \log N\right]\right\},
\end{aligned}
$$

and so

$$
\begin{aligned}
I_{2}(t) \leq & \frac{\text { const }}{N^{s+\sigma}}\left\{\frac{1}{N^{\sigma+5 / 2-s}} \int_{0}^{1}\left(\sqrt{1-x}+N^{-1}\right)^{-5 / 2}|x-t|^{v} d x\right. \\
& \left.+\log N \int_{0}^{1}\left(\sqrt{1-x}+N^{-1}\right)^{\sigma-s}|x-t|^{v} d x\right\} .
\end{aligned}
$$

Finally the assertion follows in consequence of Lemma 1.

Using a similar procedure as outlined in mentioned theorem, we have the following corollary for logarithmic kernels which we refrain from going into proof details.

Corollary 1 Let $f(x)=(1-x)^{\sigma}$ which $\sigma>0$ and not integer, then we have

$$
\int_{0}^{1}\left|f(x)-L_{N}^{*}(f ; x)\right||\log | x-t|| d x \leq \mathrm{const} \begin{cases}N^{-2-2 \sigma} \log ^{2} N, & |t| \leq 1, \\ N^{-2-2 \sigma} \log N, & 0 \leq t<1,\end{cases}
$$

where const is independent of $t$ and $N$. 
The following theorem summarizes some existing results regarding the convergence properties of the underlying product integration quadrature presented in (3.2), which is as a consequence of Theorem 2 and Corollary 1 .

Theorem 3 Let $\left\{x_{j}\right\}_{j=1}^{N}$ be distinct zeroes of the N th degree member of a set of new orthogonal polynomials on $[0,1]$ and $L_{N}^{*}(f ; x)$ denote the interpolating polynomial of degree $N$ that coincides with the function $f$ at the nodes $\left\{x_{i}\right\}_{i=1}^{N} \bigcup\{0\}$. Moreover, suppose $p(x, t)$ is a kernel of type (i) or (ii), then for every function $f$ containing only endpoints singularity, and in particular for every function $f \in C[0,1]$

$$
\lim _{N \rightarrow \infty}\left\|\int_{0}^{x} p(x, t) f(t) d t-\int_{0}^{x} p(x, t) L_{N}^{*}(f ; t) d t\right\|_{\infty}=0,
$$

moreover

$$
\begin{aligned}
& \left\|t_{N}\left(|x-t|^{-v}, f ; x\right)\right\|_{\infty}=O\left\{N^{-2-2 \sigma+2 v} \log N\right\}, \quad 0<v<1, \\
& \left\|t_{N}(\log (|x-t|, f ; x))\right\|_{\infty}=O\left\{N^{-2-2 \sigma} \log ^{2} N\right\}
\end{aligned}
$$

Proof Note that

$$
\begin{aligned}
\left|t_{N}(p(x, t), f ; x)\right| & \leq \int_{0}^{x}|p(x, t)|\left|f(t)-L_{N}^{*}(f ; t)\right| d t \\
& \leq \int_{0}^{1}|p(x, t)|\left|f(t)-L_{N}^{*}(f ; t)\right| d t .
\end{aligned}
$$

The proof follows immediately from a consequence of Theorem 2 and Corollary 1 . The bounds (4.10) and (4.11) supply an estimate of the rate of convergence.

It should be noted that our main concern is to error estimation of (4.1). The behavior of term $\left\|\left(I-A_{N}\right)^{-1}\right\|_{\infty}$ in (4.4) has been investigated under some assumptions in [21]. Based of this idea, we can establish the boundedness of the $\left\|\left(I-A_{N}\right)^{-1}\right\|_{\infty}$. (For further details see Theorem 2 of [21].)

What we have done in this section, it gives the following main result of this paper concerning the accuracy of described method.

Theorem 4 Let $f(x)$ and $f_{N}(x)$ be the exact and approximate solutions of the equation (4.1), respectively, which constructed on a set of distinct nodes $\left\{x_{i}\right\}_{i=1}^{N} \bigcup\{0\}$. If the nodes $\left\{x_{i}\right\}_{i=1}^{N}$ are the zeroes of the polynomial $P_{N 0}(x)$ which is belong to a set of new orthogonal polynomials on $[0,1]$ and $p(x, t)$ is the kernel function of the form (i) or (ii), then $f_{N}(x)$ converges uniformly to $f(x)$. Moreover the rate of convergence coincides with the product integration quadrature which we choose to approximate the integral term (4.1).

\section{Numerical Results and Discussion}

The disceretization algorithm based on Lobatto nodes (i.e. on the nodes coinciding with the zeroes of the mentioned orthogonal polynomials of the $N$ th degree in addition to the 
endpoints $x=0$ and 1) together with a product integration method described in section (3.1) has been implemented to the following test problems, taken from [5, 10, 24, 25]:

$$
\begin{aligned}
& f(x)=-\frac{1}{2} x^{2} \ln x+\frac{3}{4} x^{2}+\sqrt{x}+\int_{0}^{x} \ln |x-t| f^{2}(t) d t, \\
& f(x)=\sqrt{x} \\
& f(x)=\sqrt{x}+\frac{3}{8} \pi x^{2}-\int_{0}^{x} \frac{1}{\sqrt{t-x}} f^{3}(t) d t, \\
& f(x)=\sqrt{x} \\
& f(x)=x-e^{x} \sqrt{\pi} \operatorname{erf}(\sqrt{\pi})+\int_{0}^{x} \frac{e^{f(x)}}{\sqrt{t-x}} d t, \\
& f(x)=x, \\
& f(x)=0.25 x^{2}-0.5 x^{2} \ln x+\sqrt{x}+\int_{0}^{x} \ln |x-t|\left(x-f^{2}(t)\right) d t, \\
& f(x)=\sqrt{x} .
\end{aligned}
$$

The comparison of our numerical results in the point $x=1$ with those obtained by means of some of the methods cited in Sect. 1 is encouraging.

Equation (5.1) was solved in [24] by a method based on Navot's quadrature formula [18] for $N=10,20,40$ using transformation which is proposed in [2] and some extrapolation algorithms. In this case, the best result has the error of order $O\left(10^{-9}\right)$. A marked improvement of the proposed method in the case of $N=5$, over the method in [24] is observed in Table 1.

To investigate the convergence behavior of the present method, we plot in Figs. 1 and 2 the absolute errors of the test problems (5.2) and (5.3). The computational results have been reported in Tables 2 and 3, respectively.

Brunner et al. [5] and recently, Khater et al. [10] have been solved the equation (5.4), by piecewise polynomial collocation method and Chebyshev polynomials expansion. Reported results of them show that for $N=128$ and 64 issued maximum errors of this problem are $O\left(10^{-6}\right)$ and $O\left(10^{-9}\right)$, respectively. Looking at Table 4, we can observe an improvement of the accuracy for $N=3$ in the case of our method respect to methods in [5] and [10].

Additional numerical experiments indicate that we can achieve to good numerical results with small $N$ (e.g. with $N=2,4$ the high accurate solution is obtained), so we are not forced to solve a large system of equations in analogy of some recent numerical methods $[2,5,21$, 24] and we can achieve to solution in a short time with low computational complexity even when we face nonlinear integral equations.

Table 1 The errors for (5.1) at $x=1$

\begin{tabular}{lll}
\hline$N$ & Present method & Method in [24] \\
\hline 5 & $1.00 \times 10^{-19}$ & - \\
10 & - & $7.320 \times 10^{-4}$ \\
20 & - & $6.583 \times 10^{-8}$ \\
40 & - & $3.038 \times 10^{-9}$ \\
\hline
\end{tabular}




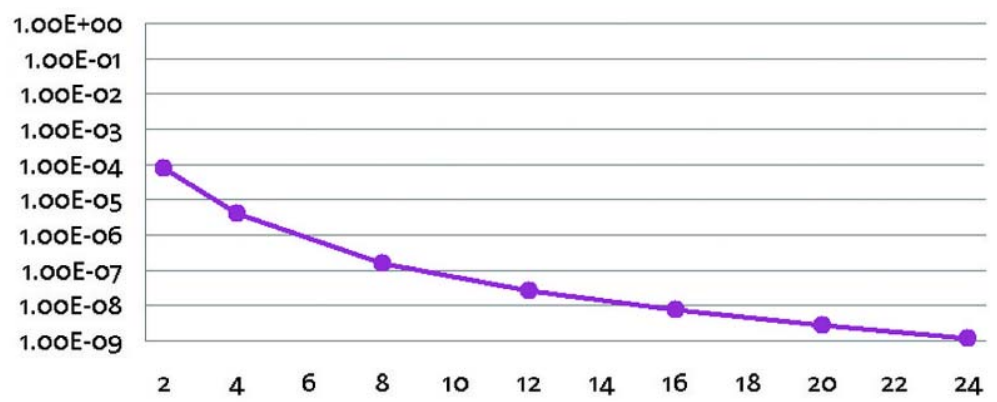

Fig. 1 The error behavior of (5.2)

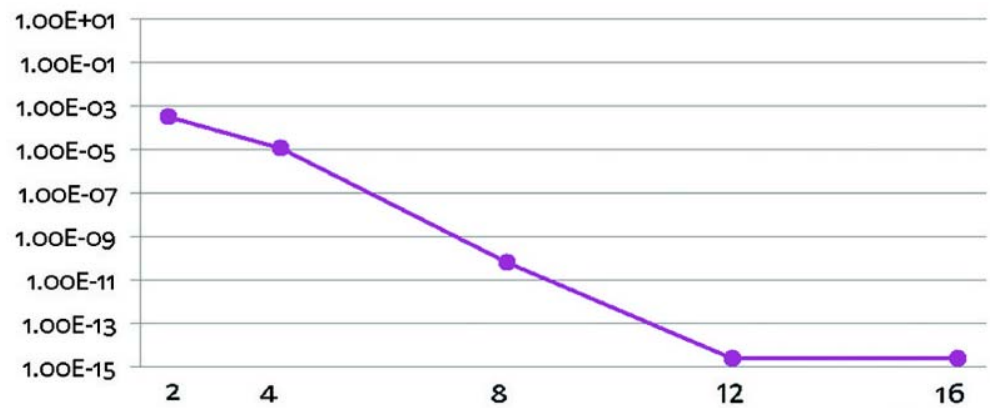

Fig. 2 The error behavior of (5.3)

Table 2 The numerical results for (5.2) at $x=1$

\begin{tabular}{lll}
\hline$N$ & Approximate solution & Absolute error \\
\hline 2 & 0.99992503150958406364 & $7.497 \times 10^{-5}$ \\
4 & 0.99999602645408519071 & $3.974 \times 10^{-6}$ \\
8 & 0.99999983926093550750 & $1.607 \times 10^{-7}$ \\
12 & 0.99999997299847153524 & $2.700 \times 10^{-8}$ \\
16 & 0.99999999247003771992 & $7.520 \times 10^{-9}$ \\
\hline
\end{tabular}

\begin{tabular}{lll}
\hline$N$ & Approximate solution & Absolute error \\
\hline 2 & 0.99998932871799920535 & $9.123 \times 10^{-4}$ \\
4 & 0.99999602645408519071 & $1.067 \times 10^{-5}$ \\
8 & 0.99999999993610453716 & $6.390 \times 10^{-11}$ \\
12 & 0.99999999999999756368 & $2.436 \times 10^{-15}$ \\
\hline
\end{tabular}

Table 3 The numerical results for (5.3) at $x=1$

\section{Conclusion}

In this paper the method of product integration based on a new family of orthogonal polynomials is used to approximate the numerical solution of nonlinear weakly singular Volterra integral equations. The new orthogonal polynomials keep distinctively of the classical or- 
Table 4 The errors for (5.4) at $x=1$

\begin{tabular}{lll}
\hline$N$ & Present method & Method in [10] \\
\hline 3 & $1.00 \times 10^{-19}$ & - \\
4 & - & $2.807 \times 10^{-4}$ \\
8 & - & $3.473 \times 10^{-5}$ \\
16 & - & $2.672 \times 10^{-6}$ \\
32 & - & $1.796 \times 10^{-7}$ \\
64 & - & $7.000 \times 10^{-9}$ \\
\hline
\end{tabular}

thogonal polynomials and give more exact quadratures. The proposed scheme can provide reasonable results for nonlinear as well as linear integral equations. Also, the method employed here can be probably extended to obtain approximate solution of Fredholm integral equations arising in various area of applied problems.

\section{References}

1. Atkinson, K.E.: An existence theorem for Abel integral equations. SIAM J. Math. Anal. 5, 729-736 (1974)

2. Baratella, P., Orsi, A.P.: A new approach to the numerical solution of weakly singular Volterra integral equations. J. Comput. Appl. Math. 163, 401-418 (2003)

3. Brunner, H.: The numerical solution of weakly singular Volterra integral equations by collocation on graded meshes. Math. Comput. 45, 417-437 (1985)

4. Brunner, H., Van der Houwen, P.J.: The Numerical Solution of Volterra Equations. North Holland, Amsterdam (1986)

5. Brunner, H., Pedas, A., Vainikko, G.: The piecewise polynomial collocation method for nonlinear weakly singular Volterra equations. Math. Comput. 67, 1079-1095 (1999)

6. Chelyshkov, V.S.: Alternative orthogonal polynomials and quadratures. Electron. Trans. Numer. Anal. 25(7), 17-26 (2006)

7. Chen, Y., Tang, T.: Convergence analysis of the Jacobi spectral collocation methods for Volterra integral equations with a weakly singular kernel (submitted for publication)

8. Griscuolo, G., Mastroianni, G., Monegato, G.: Convergence properties of a class of product formulas for weakly singular integral equations. Math. Comput. 55, 213-230 (1990)

9. Kaneko, H., Xu, Y.: Gauss-type quadratures for weakly singular integrals and their application to Fredholm integral equations of second kind. Math. Comput. 62, 739-753 (1994)

10. Khater, A.H., Shamardan, A.B., Callebaut, D.K., Sakran, M.R.A.: Solving integral equations with logarithmic kernels by Chebyshev polynomials. Numer. Algorithms 47, 81-93 (2008)

11. Keller, J.B., Olmstead, W.E.: Temperature of a nonlinear radiating semi-infinite solid. Q. Appl. Math. 29, 559-566 (1972)

12. Kershaw, D.: Some results for Volterra integral equations of second kind. In: Baker, C.T.H., Miller, G.F. (eds.) Treatment of Integral Equation by Numeric Methods, pp. 273-282. Academic Press, London (1982)

13. Krylov, V.I.: Approximate Calculation of Integrals. Macmillan Company, New York (1962)

14. Kythe, P.K., Puri, P.: Computational Methods for Linear Integral Equations. Birkhäuser, Boston (2002)

15. Levinson, N.: A nonlinear Volterra equation arising in the theory of super-fluidity. J. Math. Anal. Appl. 1, 1-11 (1960)

16. Linz, P.: Analytical and Numerical Methods for Volterra Equations. SIAM, Philadelphia (1985)

17. Monegato, G., Scuderi, L.: High order methods for weakly singular integral equations with nonsmooth input functions. Math. Comput. 67(224), 1493-1515 (1998)

18. Navot, I.: A further extension of Euler-Maclaurin summation formula. J. Math. Phys. 41, 155-184 (1962)

19. Nevai, P.: Orthogonal polynomials. Mem. Amer. Math. Soc. 213 (1979)

20. Nevai, P.: Mean convergence of Lagrange interpolation. III. Trans. Am. Math. Soc. 282, 669-698 (1984)

21. Orsi, A.P.: Product integration for Volterra integral equations of the second kind with weakly singular kernels. Math. Comput. 212, 1201-1212 (1996) 
22. Pylak, D.: Application of Jacobi polynomials to the approximate solution of a singular integral equation with Cauchy kernel on the real half-line. Comput. Method Appl. Math. 6(3), 326-335 (2006)

23. Tao, L., Yong, H.: A generalization of discrete Gronwall inequality and its application to weakly singular Volterra integral equation of second kind. J. Math. Anal. Appl. 282, 56-62 (2001)

24. Tao, L., Yong, H.: Extrapolation method for solving weakly singular nonlinear Volterra integral equations of second kind. J. Math. Anal. Appl. 324, 225-237 (2006)

25. Wazwaz, A.M., Khuri, S.A.: A reliable technique for solving the weakly singular second-kind Volterratype integral equations. Appl. Math. Comput. 80, 287-299 (1990) 